\title{
Thenar motor unit count estimates in the carpel tunnel syndrome
}

\author{
W. F. BROWN
}

From St. Joseph's Hospital, London, Ontario, Canada

SUMMARY Thenar motor unit counts were done in 19 subjects with symptoms characteristic of the carpal tunnel syndrome. Three-quarters of the patients had motor unit count estimates less than 2 SD lower limit of controls. Despite this, most did not have thenar wasting or weakness. Functional compensation by motor unit remodelling probably kept pace with motor axon loss and masked the true extent of axonal degeneration.

The carpal tunnel syndrome is common. In most cases the clinical features are sufficiently characteristic to allow a confident diagnosis to be made. Electrophysiological studies are of the most value when they provide clear evidence for a local injury to the median nerve in the carpal tunnel region, especially in patients whose symptoms and signs are unusual or indefinite. Among present methods for studying the carpal tunnel disorder, demonstration of prolonged motor terminal delays (Simpson, 1956; Thomas, Lambert, and Cseuz, 1967) and abnormal median digital sensory nerve potentials (Thomas et al., 1967) are the most useful. Electromyography may provide evidence of denervation of the thenar muscles. In one study, however, fibrillation potentials were found in only $41 \%$ of cases (Thomas et al., 1967) and even less frequently in another report (Buchthal and Rosenfalck, 1971). A recent report clearly demonstrated abnormal sensory fibres conduction across the carpal tunnel despite, in many subjects, normal conventional sensory potentials and distal motor latencies (Buchthal and Rosenfalck, 1971). Measurement of the transtunnel sensory conduction velocity required, however, special precautions and recording techniques.

These methods, unfortunately, do not provide more than a rough estimate of the extent of median nerve fibre degeneration. The present study was undertaken to see if an estimate of the thenar motor unit count was helpful in demon- strating denervation by excessive motor unit loss, especially in those subjects with mild carpal tunnel syndromes, without weakness or thenar wasting.

\section{METHODS}

SUBJECTS The study was limited to a group of $\overrightarrow{0}$ subjects, 10 of whom were female, with symptoms $\omega$ characteristic of the carpal tunnel syndrome. All subjects were troubled, many times, during the night and on getting up in the morning, with numbness and prickliness in the thumb and next three fingers. The symptoms were often most severe on wakening in the morning and, in some, were aggravated by use of the hands during the day. None had other limb symptoms or signs to suggest a cervical root or other peripheral nerve injury.

In five there was mild weakness of thumb abduction but no wasting and, in two others, obvious wasting and severe weakness. In the rest, the thenaro strength and mass were normal. In all subjects the assessment of strength and the presence or absence of wasting were based on the statement of at least twoô neurological consultants who reached their opinions independently. If their reports differed, as they did in윽 two subjects, the more abnormal estimate was accepted.

ELECTROPHYSIOLOGICAL STUDIES A method for estimating the number of motor units in thenaro muscles has been described (Brown, 1972). For thew present study, the Devices Model 3072 high voltage stimulator was modified by replacing the standardo single turn $5 \mathrm{k} \Omega$ wirewound output potentiometere 194 


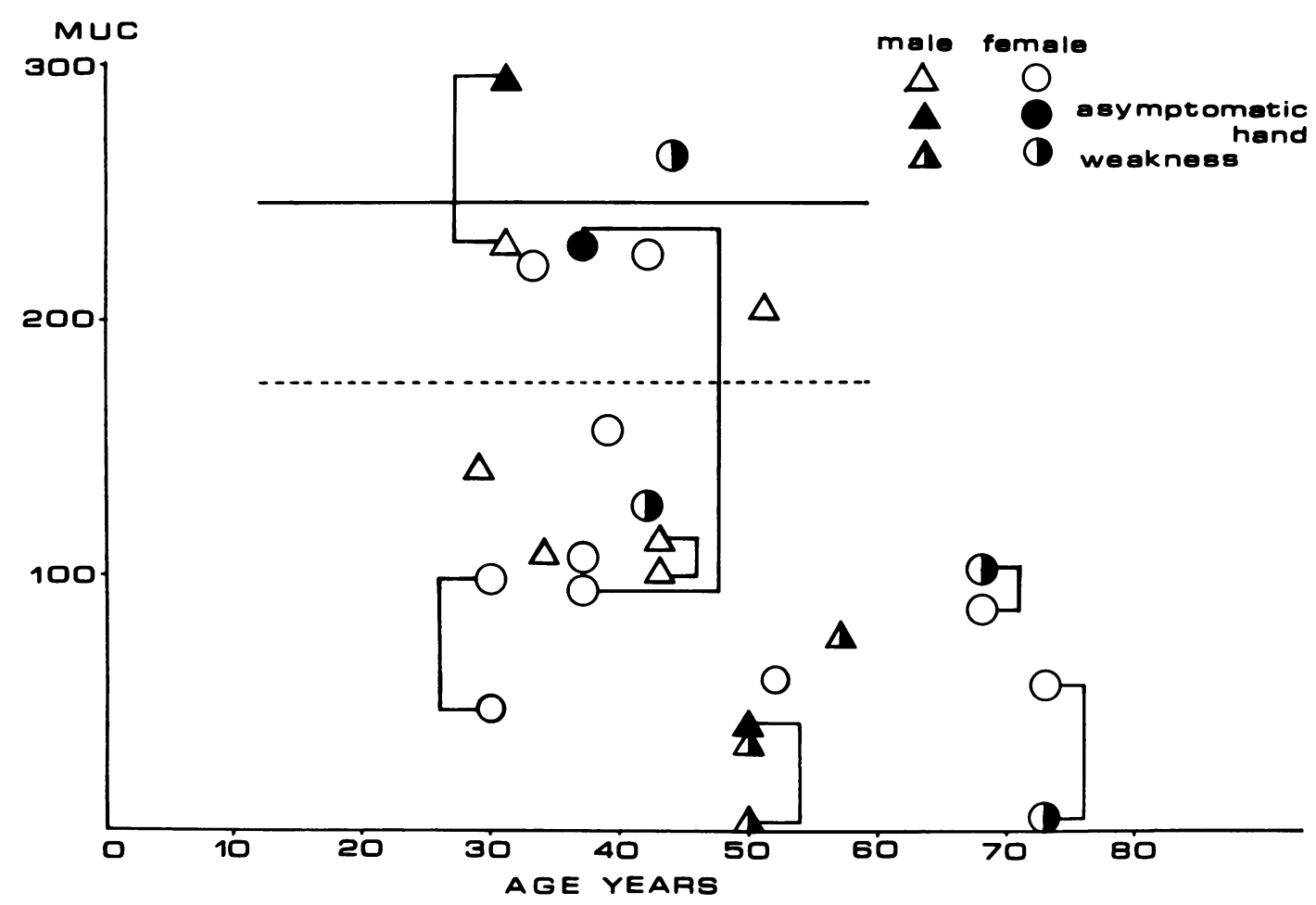

FIG. 1. Plot of the motor unit count of all 19 subjects with a clinical diagnosis of the carpal tunnel syndrome. Also shown are the mean and $2 S D$ lower limit of all controls under 60 years of age. The vertical bar connects values in both hands of the same subject.

with a 10 turn potentiometer. This modification did not alter the motor unit estimates but did make it easier to separate individual motor unit thresholds.

Measurements were made of the terminal delays to the first several motor unit potential steps, as well as to the maximum compound action potential. In all subjects, a careful search for spontaneous fibrillation potentials in the thenar muscles was made.

Sensory nerve action potential studies were done in 15 of the subjects by using needle electrodes as described by Buchthal and Rosenfalck (1966).

\section{RESULTS}

In Fig. 1 the motor unit counts in all 19 carpal tunnel subjects are plotted against age. For comparison, the mean and 2 SD lower limit of all controls under 60 years of age reported previously are shown (Brown, 1972). There were five subjects with motor unit counts within the $2 \mathrm{SD}$ limits of the control mean. Under 60 years of age, 11 subjects had motor unit counts less than the $2 \mathrm{SD}$ below the lower limit and eight of these were judged to have no thenar wasting or weakness. For example, a 30 year old female shown in Fig. 2 had typical sensory symptoms in the right hand for two years and in the left for nine months, but no weakness or wasting in either hand. In both hands the motor unit count was reduced.

In another subject, the asymptomatic hand shown in Fig. 3 also had a prolonged terminal motor delay and clearly reduced motor unit count, but normal power. However, in the symptomatic hand, abduction was very weak, the thenar muscles obviously wasted and only 3 motor units could be evoked with supramaximal median nerve stimulus. Fibrillation potentials were not present in either hand.

Only two subjects had severe wasting and weakness and both had fewer than 5 motor units 

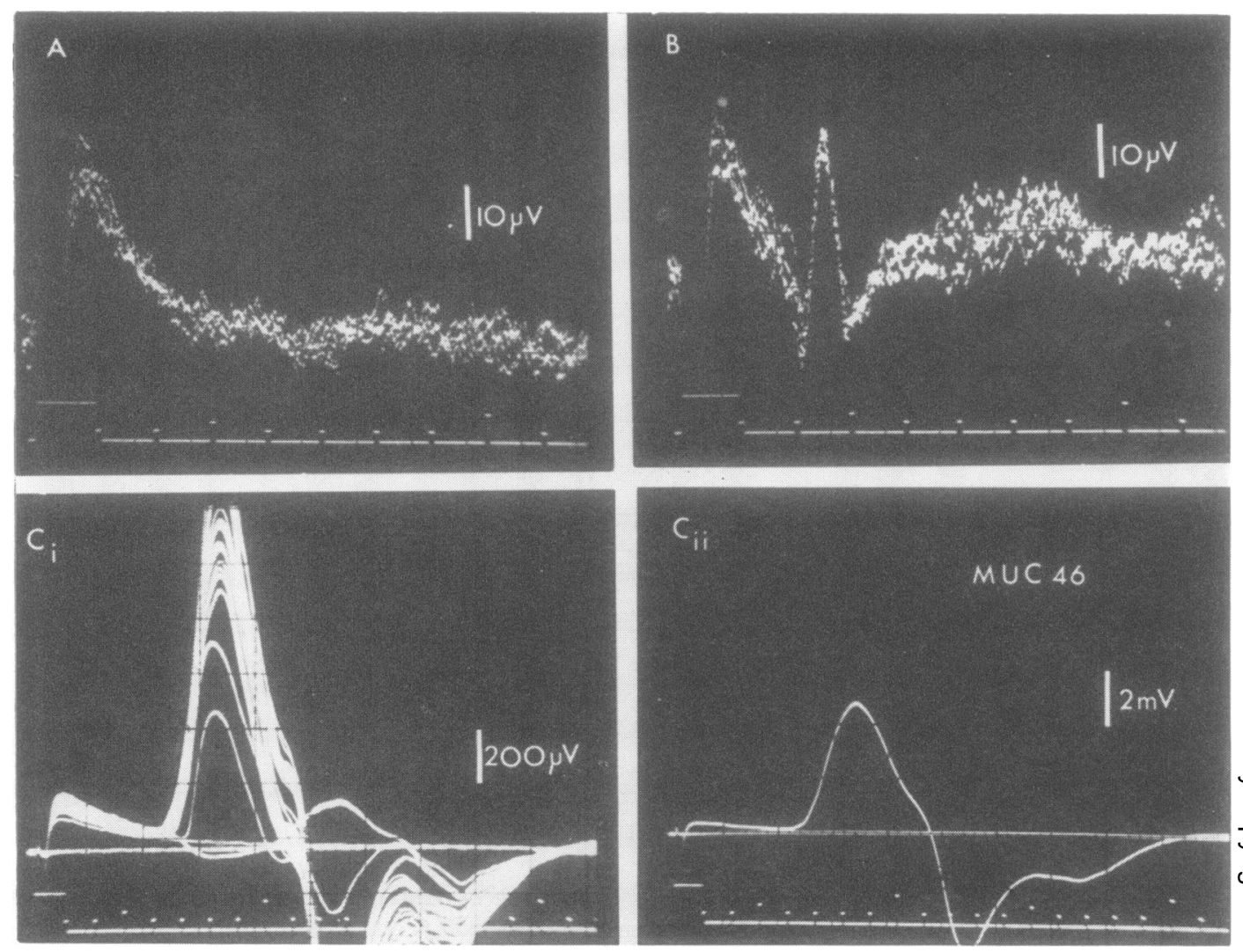

FIG. 2. A 30 year old female with bilateral sensory symptoms but no wasting or weakness of the thenar muscles. Right hand, A: thumb median nerve sensory potential; B: 4th and 5th finger ulnar nerve sensory potential. Motor unit estimate-99. Left hand, Ci-ii: motor unit estimate-46.

estimated in thenar muscles. The lowest motor unit count, in both control and carpal tunnel groups, without wasting or weakness, was 40 . In one subject, reported to have mild weakness of thumb abduction but without wasting, the motor unit count was in the normal range. Unfortunately, a motor unit count was not done in the opposite hand for comparison.

Motor unit counts were done of both hands in six subjects. In two of these the symptoms were in one hand only and in both the motor unit count was lower in the hand with symptoms. The motor unit count in both hands was within the normal range in one individual but the value was $20 \%$ lower in the hand with symptoms.

Only two patients of the carpal tunnel group were found to have fibrillation potentials. In both, there was mild weakness and the motor unit count in thenar muscles was reduced.

In the control group there were only three cases with motor terminal delays over $4.7 \mathrm{msec}$ ? and two of these were in subjects over 80 years $\frac{5}{3}$ of age (mean $3.8 \pm 0.6 \mathrm{msec}$ ). In the carpal tunnel group, half of the subjects had terminal motor delays greater than $4.7 \mathrm{msec}$ (mean $4.8 \pm 1.0 \mathrm{msec}$ ). The longest terminal motor delay in the carpal tunnel group was $7.3 \mathrm{msec}$. The mean maximum motor conduction velocity in the forearm in carpal tunnel subjects was $55 \pm \sigma$ $1 \cdot 2 \mathrm{~m} / \mathrm{sec}$ compared with the control group values $N$ of $56 \pm 4 \cdot 9$.

The median digital sensory nerve action 0 potentials recorded just above the wrist were 0 abnormal in nine of the 15 subjects studied. 

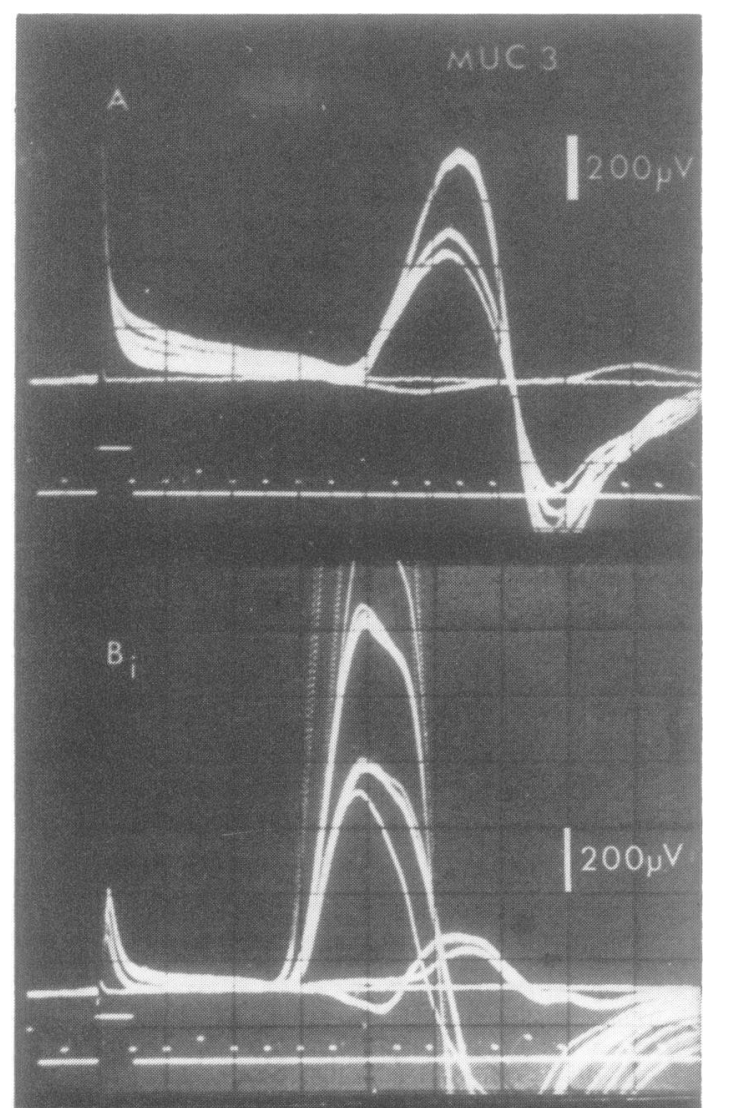

MUC 40

B.

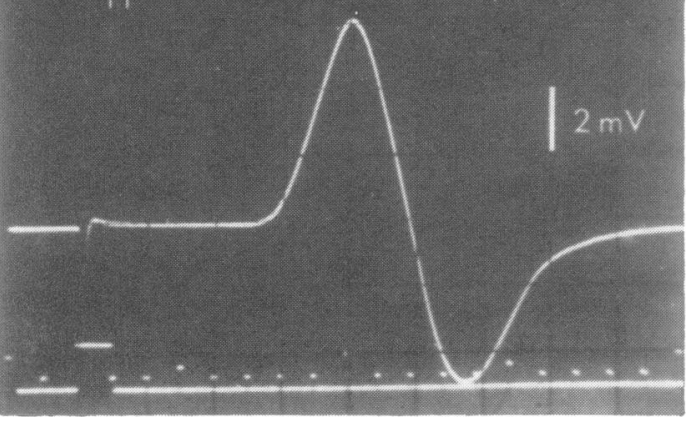

FIG. 3. A fifty year old male with unilateral sensory symptoms, severe wasting and weakness. A: motor unit count in symptomatic hand shows only 3 motor unit steps with a supramaximal stimulus. B $i-i i$ : motor unit count in asymptomatic hand without wasting or weakness. Motor unit count-40.

\section{DISCUSSION}

Clinical estimates of strength and muscle mass are difficult to quantitate. Their accuracy depends on the training and experience of the clinician. Whatever criticisms can be made of the estimates of muscle strength and mass, their value is to provide a standard against which the neurophysiological estimates of the motor unit counts can be made. Judged in this light, the motor unit count was found to be of value because in several subjects judged normal on clinical examination and in whom no fibrillation potentials could be detected, the estimated motor unit count was clearly lower than in the control values of comparable ages.

The motor unit count assumes that all motor axons are activated by the supramaximal nerve stimulus. Further, the motor axon action potentials are assumed to propagate without distal failure or block. Failure to activate all of the motor axons by the stimulus or axonal block beyond the stimulus point could result in falsely low motor unit counts.

A single necropsy study of one patient with signs and symptoms typical of the carpal tunnel syndrome was reported by Thomas and Fullerton (1963). These authors showed that the nerve fibre size was sharply reduced under the flexor retinaculum and that the nerve fibres distal to the carpal tunnel were consistently larger. In one hand there was gross weakness and wasting of the abductor pollicis brevis muscle and a very prolonged terminal motor delay. The nerve fibre count in the carpal tunnel region was one-third fewer than the number at the mid forearm level counted in a standard strip across the widest diameter of the nerve section.

Fullerton and Gilliatt (1967) described a local injury to the median and ulnar nerves under a cartilaginous bar in the guinea-pig. The pathological features included not only Wallerian degeneration but many fibres, in addition, showed thinly myelinated internodes and others complete myelin loss. Intermittent conduction block was clearly shown in demyelinated single fibres of the rat, especially in those fibres with greatly prolonged internode conduction times (Rasminsky and Sears, 1971).

There was no suggestion in this study of intermittent block of single motor unit poten- 
tials, even when the terminal motor delay was prolonged. The absence of intermittent single unit block and the very prolonged terminal motor delays reported in other series (Simpson, 1956; Thomas, 1960; and Thomas et al., 1967) made it unlikely that a functional axonal block accounted for the lower motor unit counts. Axonal degeneration, therefore, probably accounted fully for the lower motor unit count, especially in those subjects with normal or near normal motor terminal delays.

The motor unit count record showed little evidence of abnormal dispersion of motor unit terminal delays, even in those subjects with abnormally prolonged terminal delays. These observations were, however, based on the first several motor unit steps and may not have represented possible wider dispersions of terminal delays in units with higher thresholds.

Unlike the present study, previous reports have shown a significant, although mild, slowing of forearm median nerve maximum motor conduction velocity in the carpal tunnel syndrome (Thomas, 1960; Thomas et al., 1967). It is difficult to compare independent studies but in both previously reported series, one-quarter to one-third of the subjects had terminal motor delays greater than the highest value reported here. Thomas (1960) showed that the magnitude of slowing of forearm conduction was related to the degree of distal slowing. The absence of such a large proportion of patients with very long terminal motor delays may therefore have accounted for the failure to show any slowing of the forearm motor conduction velocities in this study. In fact, based on the absence of very prolonged terminal motor delays, normal median forearm motor conduction velocities and the relative infrequency of fibrillation potentials, it is possible that the present study included a higher proportion of mildly affected subjects than previous reports.

It was surprising from the motor unit studies of ageing and the carpal tunnel syndrome, how few motor units were required to maintain muscle size and strength. McComas has previously reported that there was no significant fall in the maximum twitch potential of the extensor digitorum brevis muscle unless the motor unit count was less than $10 \%$ of the normal value (McComas, Sica, Campbell, and Upton, 1971).

In the present study, weakness and wasting, unless severe, was not a useful guide to the extent of motor unit loss except when the nerve fibre loss had been severe. Functional compensation by constant remodelling of motor units probably masked the true extent of motor fibre axonal degeneration. Weakness and wasting, if present, may suggest that the major proportion of the axonal injury took place over a short period of time and not scattered over many months or years, where remodelling is more likely to mask the motor axon loss.

The author would like to thank Dr. H. Barnett, Dr. H. Barr, Dr. J. Girvin, and Dr. N. Jaatoul for providing me with their clinical notes and patients for $\omega$ the study. I am most grateful for the able and patient is assistance of Mrs. M. Webster in the preparation of $\vec{\varphi}$ this manuscript.

\section{REFERENCES}

Brown, W. F. (1972). A method for estimating the number motor units in the thenar muscles and the changes in mote unit count with ageing. Journal of Neurology, Neurs surgery, and Psychiatry, 35, 845-852.

Buchthal, F., and Rosenfalck, A. (1966). Evoked action potentials and conduction velocity in human sensory nerves. Brain Research, 3, 1-122.

Buchthal, F., and Rosenfalck, A. (1971). Sensory conduction from digit to palm and from palm to wrist in the carpal tunnel syndrome. Journal of Neurology, Neurosurgery, and Psychiatry, 34, 243-252.

Fullerton, P. M., and Gilliatt, R. W. (1967). Median and ulnar neuropathy in the guinea-pig. Journal of Neurology, Neurosurgery, and Psychiatry, 30, 393-402.

McComas, A. J., Sica, R. E. P., Campbell, M. J., and Upton, A. R. M. (1971). Functional compensation in partially denervated muscles. Journal of Neurology, Neurosurgery, and Psychiatry, 34, 453-460.

Rasminsky, M., and Sears, T. A. (1971). Conduction time between nodes of Ranvier in normal and demyelinated single fibres. Abstract, Fourth International Congress of 3 . Electromyography, Brussels.

Simpson, J. A. (1956). Electrical signs in the diagnosis of carpal tunnel and related syndromes. Journal of Neurology, Neurosurgery, and Psychiatry, 19, 275-280.

Thomas, P. K. (1960). Motor nerve conduction in the carpal 을 tunnel syndrome. Neurology (Minneapolis), 10, 1045-1050. D

Thomas, P. K., and Fullerton, P. M. (1963). Nerve fibre size으․ in the carpal tunnel syndrome. Journal of Neurology, Neurosurgery, and Psychiatry, 26, 520-527.

Thomas, J. E., Lambert, E. H., and Cseuz, K. A. (1967). Electrodiagnostic aspects of the carpal tunnel syndrome. Archives of Neurology, 16, 635-641. 